\title{
Pentaquark Update After Ten Years
}

\author{
Harry J. Lipkin ${ }^{a, b *}$ \\ ${ }^{a}$ Department of Particle Physics \\ Weizmann Institute of Science, Rehovot 76100, Israel \\ ${ }^{b}$ School of Physics and Astronomy \\ Raymond and Beverly Sackler Faculty of Exact Sciences \\ Tel Aviv University, Tel Aviv, Israel
}

\begin{abstract}
Studying scattering of heavy flavor hadrons and looking for bound states is shown to give experimental information otherwise unobtainable about effective two-body interactions between constituent $(q q)_{6}$ and $(\bar{q} q)_{8}$ pairs respectively in color sextet and color octet states. All the successes of the constituent quark model in ( $u d s$ ) hadron spectroscopy are shown to depend only on effective two-body interactions in color $3^{*}$ and singlet states.

New directions for bound pentaquark searches are discussed following the availability of vertex detectors which can pinpoint events where a proton is emitted from a secondary vertex. Any such event indicates a particle decaying weakly by proton emission and the discovery of a new particle if its mass is higher than that of known charmed baryons. There is no combinatorial background and striking decay signatures like $p \phi \pi^{-}$are no longer needed.

The beauty pentaquark ( $\bar{b} s u u d)$ and the doubly-strange pentaquark $(\bar{c} s s u d)$ may be relevant to future searches. A simple calculation shows that the effects of flavor-SU(3) breaking on their binding relative to the relevant thresholds are similar to that for the singly-strange pentaquark ( $\bar{c} s u u d)$ relative to the $D_{s} p$ threshold.
\end{abstract}

\section{INTRODUCTION}

It is now ten years since the proposal of the existence of the pentaquark [1] and suggestions for its search via the $p \phi \pi^{-}$decay mode [2] [ Despite subsequent experimental

*Supported in part by grant No. I-0304-120-.07/93 from The German-Israeli Foundation for Scientific Research and Development 
progress and analysis of models for pentaquark structure, decay and signatures [9] there is today still no convincing experimental evidence for the existence of the pentaquark nor of any other exotic hadron which cannot be described in a constituent quark model as a $3 q$ or $\bar{q} q$ state [10,11]. Not a single theoretical prediction for the multiquark sector has been confirmed by experiment [8,12].

Why should anyone look for an anticharmed strange baryon? Why should it be bound? Who cares whether it is bound? In trying to understand how QCD makes hadrons out of quarks and gluons, we note that long-standing regularities and paradoxes in simple experimental hadron physics still remain to be explained by QCD. Today the simple LevinFrankfurt additive quark model prediction [13,14] still fits experimental data up to 310 Gev/c [16] with a discrepancy always less than $7 \%$ for the prediction

$$
\delta_{A Q M} \equiv(2 / 3) \cdot \sigma_{t o t}(p p)-\sigma_{t o t}\left(\pi^{-} p\right) \approx 0
$$

There seems to be underlying dynamics describing the meson-baryon difference primarily in terms of the number of constituent quarks. The pion is still $2 / 3$ of a proton even though some theorists say that the pion is a Goldstone boson and the proton is a skyrmion. Further evidence that mesons and baryons are made of the same quarks is given by the remarkable successes of the constituent quark model, in which static properties and low lying excitations of both mesons and baryons are described as simple composites of asymptotically free quasiparticles having an effective mass with exactly the same value for predicting hadron masses, magnetic moments and hyperfine splittings.

The mass difference between strange and nonstrange quarks $m_{s}-m_{u}$ is found to have the same value $\pm 3 \%$ when calculated in two independent ways from baryon masses and in two independent ways from meson masses [17 [19],

$$
\begin{gathered}
\left\langle m_{s}-m_{u}\right\rangle_{\text {Bar }}=M_{\Lambda}-M_{N}=177 \mathrm{MeV}=\frac{M_{N}+M_{\Delta}}{6} \cdot\left(\frac{M_{\Delta}-M_{N}}{M_{\Sigma^{*}}-M_{\Sigma}}-1\right)=190 \mathrm{MeV} . \\
\left\langle m_{s}-m_{u}\right\rangle_{m e s}=\frac{3\left(M_{K^{*}}-M_{\rho}\right)+M_{K}-M_{\pi}}{4}=180 \mathrm{MeV}=
\end{gathered}
$$




$$
=\frac{3 M_{\rho}+M_{\pi}}{8} \cdot\left(\frac{M_{\rho}-M_{\pi}}{M_{K^{*}}-M_{K}}-1\right)=178 \mathrm{MeV} .
$$

The same approach applied to the mass difference between $b$ and $c$ quarks $m_{b}-m_{c}$ gives

$$
\begin{array}{r}
\left\langle m_{b}-m_{c}\right\rangle_{\text {Bar }}=M\left(\Lambda_{b}\right)-M\left(\Lambda_{c}\right)=3356 \mathrm{MeV} \\
\left\langle m_{b}-m_{c}\right\rangle_{m e s}=\frac{3\left(M_{B^{*}}-M_{D^{*}}\right)+M_{B}-M_{D}}{4}=3338 \mathrm{MeV} .
\end{array}
$$

The ratio $\frac{m_{s}}{m_{u}}$ has the same value $\pm 2.5 \%$ for mesons and baryons.

$$
\left(\frac{m_{s}}{m_{u}}\right)_{\text {Bar }}=\frac{M_{\Delta}-M_{N}}{M_{\Sigma^{*}}-M_{\Sigma}}=1.53=\left(\frac{m_{s}}{m_{u}}\right)_{M e s}=\frac{M_{\rho}-M_{\pi}}{M_{K^{*}}-M_{K}}=1.61
$$

We also note three predictions of hadron magnetic moments with no free parameters [20,21]

$$
\begin{gathered}
\mu_{\Lambda}=-0.61 \text { n.m. }=-\frac{\mu_{p}}{3} \cdot \frac{m_{u}}{m_{s}}=-\frac{\mu_{p}}{3} \frac{M_{\Sigma^{*}}-M_{\Sigma}}{M_{\Delta}-M_{N}}=-0.61 \mathrm{n} . \mathrm{m} . \\
-1.46=\frac{\mu_{p}}{\mu_{n}}=-\frac{3}{2} \\
\mu_{p}+\mu_{n}=0.88 \text { n.m. }=\frac{M_{p}}{3 m_{u}}=\frac{2 M_{p}}{M_{N}+M_{\Delta}}=0.865 \text { n.m. }
\end{gathered}
$$

As long as QCD calculations have not yet succeeded to explain these striking experimental facts, it is of interest to search for new experimental input.

Additional input is obtainable from investigations of two striking features of the hadron spectrum: (1) the absence of strongly bound multiquark exotic states like a dipion with a mass less than two pion masses or a dibaryon bound by $100 \mathrm{MeV}$; (2) the structure of nuclei as composed of three-quark clusters called nucleons rather than a quark gas, quark bags, a quark shell model [22,23] or a quark-gluon plasma. The constituent quark model gives a very simple answer [24]. But the validity of this simple picture still remains to be confirmed by experiment.

All the successes of the constituent quark model with a two-body color-exchange interaction [25,20,18, 19] and all of hadron spectroscopy without exotics including scattering still give information only about the $(\bar{q} q)_{1}$ interaction in the color singlet state and the $(q q)_{3 *}$ interaction in the color antitriplet state and no information about short-range $(q q)_{6}$ or $(\bar{q} q)_{8}$ 
interactions in color sextet or color octet states. The two-body color-exchange color-electric interaction commonly used saturates [25] and gives no forces between two color singlet hadrons. The two-body color-exchange color-magnetic interaction is always repulsive between two quarks of the same flavor. Thus baryon-nucleon scattering in the $(\mathrm{u}, \mathrm{d}, \mathrm{s})$ sector is dominated by a short-range repulsion (the well-known repulsive core in the nucleon-nucleon interaction). Meson-hadron scattering in the (uds) sector must have either a quark or an antiquark in the meson with the same flavor as one of the quarks in the other hadron. Two quarks of the same flavor have a repulsive color-magnetic interaction keeping the two hadrons apart. A $\bar{q} q$ pair of the same flavor can annihilate and produce a hadron resonance. Thus hadron-hadron scattering in the $(\mathrm{u}, \mathrm{d}, \mathrm{s})$ sector is dominated either by $q q$ repulsion or by resonances produced by $\bar{q} q$ annihilation.

Only when there are more than three flavors is it possible to have realistic scattering experiments (unrealistic cases like $K^{-} \Delta^{-}$and $\phi N$ are excluded) in which there is no common flavor between beam and target and the $(q q)_{6}$ or $(\bar{q} q)_{8}$ interactions can be observed.

The possible existence of exotic hadrons remains a principal question in hadron spectroscopy and the understanding of how the binding of quarks and gluons into hadrons is described by QCD [12]. The first exotic hadron search was for the $H$ dibaryon [26]. Jaffe's original calculation and subsequent work 27] indicate a gain in hyperfine interaction energy by recoupling color and spins in the six quark system over the two- $\Lambda$ system. But a lattice gauge calculation [28] indicated the $H$ to be unbound and well above the $\Lambda \Lambda$ threshold. The lattice calculation showed a repulsive $\Lambda$ - $\Lambda$ interaction generated by quark exchange [7,29 which is not included in simple model calculations and could well prevent the six quarks from coming close enough together to feel the additional binding of the short range hyperfine interaction.

Such a repulsive exchange force can not be present in pentaquarks, which were shown to have a hyperfine binding roughly equal to that of the $H$, but with no possible quark exchange force in the lowest decay channel $D_{s} N$ [7]. The simplest lattice calculation with an infinitely heavy charmed antiquark and four light quarks uuds, can easily be done in 
parallel with the more complicated $\mathrm{H}$ calculation both in the symmetry limit where all light quarks have the same mass and with $S U(3)$ symmetry breaking. Comparing the results for these cases may provide considerable insight into our understanding of the physics of QCD in multiquark systems even if the pentaquark is not found as a physical bound state in experiment. There is therefore interest both in experimental searches for the pentaquark and in lattice gauge calculations. However, so far no such lattice calculation has been done or is planned.

The color-magnetic interaction can also give a molecular-type wave function extending over a distance large compared with the range of the hyperfine interaction. Such models have been proposed for the $a_{o}$ and $f_{o}$ mesons [30,31]. A rough estimate of the binding [31]. indicates that if the $a_{o}$ and $f_{o}$ are really barely bound $K \bar{K}$ molecular states [30, 31] the $H$ and Pentaquark should be more strongly bound [5] and be excellent candidates for weakly bound molecular states.

\section{HOW GOOD VERTEX DETECTORS CHANGE SIGNATURE REQUIREMENTS}

\section{A. Only background is wrongly-measured events}

Without vertex detectors a pentaquark decay signal appears against a large combinatorial background. Peaks in a mass spectrum can arise from statistical fluctuations in the background. Standard statistical considerations are therefore needed to analyze data.

With good vertex detectors which can distinguish between particles originating from primary and secondary vertices, every event in which a decay proton is observed coming from a secondary vertex is evidence for a particle which decays weakly by proton emission. If its mass is not consistent with the mass of a known weakly decaying baryon, the event is either evidence for a new as yet unknown particle or it is a wrong event incorrectly measured. It cannot be a statistical fluctuation of known physics if measured correctly. Thus 
an experiment which can clearly distinguish a proton coming definitely from a secondary vertex should be considered as an open search for new weakly decaying baryons and not simply as a pentaquark search. One might even find new physics beyond the standard model. This more general framework should be used both in planning experiments and in analyzing data from ongoing experiments, rather than considering only the pentaquark. This point has not been noted in previous articles on pentaquark searches [9, 32

Once data are obtained and events remain which pass all cuts indicating that they have a proton emitted from a secondary vertex, the main question is not whether this is sufficient evidence for the pentaquark. It is rather whether these events are real and indicate evidence for a new weakly decaying baryon, or whether they are somehow incorrectly measured. One must investigate all possibilities of incorrect measurement.

\section{B. The Beauty Pentaquark $P_{\bar{b} s u u d}$}

The same considerations apply to baryons containing $b$ quarks, where every event in which a decay proton is observed coming from a secondary vertex may also indicate the presence of a new particle. The calculations indicating that the charmed pentaquark may be bound apply also for analogous states with heavier antiquarks; e.g. the beauty pentaquark $P_{\bar{b} \text { suud }}$ which are equally attractive candidates for bound exotics. Many such calculations

can be used directly for the beauty pentaquark by simply replacing the $\bar{c}$ everywhere by $\bar{b}$. The lowest threshold here is $p B_{s}$ and many of the same analyses of the $p D_{s}$ system used for the charmed pentaquark can also be taken over directly here, including the molecular model in which an off-shell $B_{s}$ is bound to a proton. There is, however one essential new ingredient; namely the existence of two dominant decay modes for the $b$ quark; namely $b \rightarrow c \bar{u} d$ and $b \rightarrow c \bar{c} s$. The second can give rise to striking signatures in which a proton and a charmonium are emitted from the same secondary vertex; e.g. $P_{\bar{b} \text { suud }} \rightarrow p \phi \psi$. Note that in this decay the mass of the $\phi \psi$ system is below the $B_{s}$ mass and cannot arise from $B_{s}$ decay. 


\section{Tests to distinguish wrongly-measured events}

The mass spectrum offers interesting clues. An anticharmed strange baryon with a mass above the $D_{s}-p$ threshold will decay strongly to $D_{s}-p$ in a primary vertex and not be observed at the secondary vertex. Thus all events in which a secondary proton is observed but which have a mass above the $D_{s}-p$ threshold must be considered as wrong events or really exotic new physics. The presence of such events which pass all cuts would probably indicate an unknown systematic error that introduces spurious events without secondary vertex protons,

A real new weakly-decaying baryon should not only produce a peak in the mass spectrum at the particle mass, but also a tail below the peak corresponding to decays in which one or more neutral particles have escaped detection. Since any weakly-interacting particle also has semileptonic decays in the standard model, events in the tail containing a muon or electron should be be expected. These are particularly significant since they confirm the identification of a weakly decaying particle.

The original suggestion directing the search to particular decay modes like $p \phi \pi^{-}$arose from the necessity to find a signal against a large statistical combinatorial background. The strategy can be quite different when the only background is wrongly measured events. One might first try an extremely stringent cut on the proton to ensure that it really is a proton that comes from a secondary vertex. This would include events with all possible numbers of prongs. One can then separate these into events with even and odd numbers of prongs, which correspond respectively to decays of neutral and charged particles. A charged pentaquark with the structure of a $D_{s}$ bound to a neutron rather than a proton would be more apt to decay to a final state containing a neutron rather than a proton, unless the final state baryon is a $\Delta^{o}$ or $N^{* o}$ decaying to $p \pi^{-}$. This immediately suggests looking for $p \pi^{-}$resonances in all odd prong events.

The quasi-two-body events now become of interest. The $p \pi^{-}$decay mode, for example would be an impossible signature without vertex detectors. But once the proton which 
definitely comes from a secondary vertex is selected, the unique energies of the particles provide a striking signal for the new particle. Even if the efficiency for selecting a secondary proton is only $5 \%$; i.e. only one out of every 20 events is really a secondary proton, the selection of a proton with an energy near the value for a $p \pi^{-}$decay would enhance this signal/noise ratio by a considerable factor. One would also expect the background from single pions apparently from the same vertex and having the right energy to be much smaller than the normal combinatorial background from multiparticle events.

If at this stage the background of presumably wrongly-measured events is still too large, the next step is to introduce cuts which hopefully eliminate wrong events without excessively reducing good events. The effectiveness of a cut can be estimated by examining the ratio of the events above the $D_{s}-p$ threshold to those below the threshold.

If cuts finally eliminate all events above the $D_{s}-p$ threshold and others below the threshold remain, the question arises what they might be. If they are all concentrated in the same mass bin, they might be considered as evidence for a new particle. If they are scattered over the mass spectrum, they must be due to some systematic error which has not been eliminated by cuts. There are also possibilities of a proton from the decay of a known charmed baryon combined with other particles from a different vertex. There can also be events from the same new particle with a lower mass because of a decay mode with an unobserved photon or $\pi^{o}$, or a semileptonic decay with an unobserved neutrino and a muon misidentified as a pion.

A real puzzle arises if several events in a single mass bin are observed and several other events with masses definitely different but too close to the other mass to be due to the same particle with a missing $\pi^{o}$. If the events are not real correctly measured events, the question arises why they only are found in a very small mass range. If they are all real, they indicate too many new particles, or some new multiplet not anticipated by theorists.

The ultimate conclusion if a small number of events are found which are not yet sufficient evidence for a new particle, but cannot be easily dismissed as due to known systematics, is that more data are needed 10 . 


\section{Lifetime Considerations}

It is not obvious how the lifetime of the pentaquark may differ from the $D_{s}$ lifetime. An off-shell $D_{s}$ bound to a proton would have a longer lifetime because the transition matrix elements would be essentially the same, but phase space would be smaller. A more complicated wave function can have factors working in both directions. There can be color factors inhibiting decay into a color-singlet baryon and several mesons. There can be new channels opening that can enhance the decay rate.

However, if the lifetime of the Pentaquark is longer than that of a charmed meson, another search criterion is opened. One can consider only events which have a delay greater than several $D_{s}$ lifetimes. If, for example, the lifetime of the pentaquark is longer than the $D_{s}$ lifetime by a factor of 2 , the choice of a minimum delay which reduces the Pentaquark signal by a factor of 10 will reduce background by a factor of 100 .

\section{THE DOUBLY-STRANGE AND BEAUTY PENTAQUARKS}

The doubly strange $P_{\bar{c} s s u d}$ is degenerate with the singly strange $P_{\bar{c} s u u d}$ in the SU(3) flavor symmetry limit and the two states have equal binding energies relative to the relevant $\Lambda D_{s}$ and $N D_{s}$ thresholds. The effects of $\mathrm{SU}(3)$ symmetry breaking have been investigated in detail for the $P_{\bar{c} \text { suud }}$ in the limit where the charmed quark has infinite mass and its hyperfine interaction energy is neglected. This limit should be an even better approximation for the beauty quark. We therefore treat both pentaquarks on the same footing in the remainder of this paper and use the notation $P_{\bar{Q} \text { suud }}$ and $P_{\bar{Q} s s u d}$ for both, where $Q$ denotes either a $c$ or $b$ quark. We now generalize the treatment of $\mathrm{SU}(3)$ breaking in this limit to apply to both singly and doubly strange pentaquarks and show that all states have very similar properties and that all may well be bound.

The stability against breakup of an exotic multiquark system has been examined by

checking whether hyperfine energy can be gained by recoupling the color and spins of the 
lowest lying two-hadron threshold [33]. A variational approach has been used with a wave function in which the two-body density matrix is the same for all pairs as in a baryon, and the experimental $N-\Delta$ mass splitting is used to determine the strength of the hyperfine interaction energy [33].

In our approximation we disregard the heavy antiquark and its small hyperfine interaction and consider only the classification of the state of the four quarks coupled to spin zero and a color triplet. These four quarks in all pentaquark states considered here contain one quark pair or "diquark" with the same flavor and one pair with a different flavor. We use the notation $\left|d_{21}^{S}\right\rangle$ and $\left|d_{15}^{S}\right\rangle$ to denote these quark pair or diquark states with spin $S$ classified in the symmetric 21 and the antisymmetric 15 dimensional representations of the color-spin SU(6). The Pauli principle requires the quark pair of the same flavor to be coupled to the antisymmetric 15. We now define the classification of the states under consideration in a conventional notation $\left|D_{6}, D_{3}, S, N\right\rangle$ 34, 355 where $D_{6}$ and $D_{3}$ denote the dimensions of the color-spin $S U(6)$ and color $\mathrm{SU}(3)$ representations in which the multiquark states are classified, $S$ and $N$ denote the total spin and the number of quarks in the system and primes distinguish between different representations with the same dimension.

$$
\begin{aligned}
|N\rangle & =|70,1,1 / 2,3\rangle \\
|\Delta\rangle & =|20,1,3 / 2,3\rangle \\
|\Lambda\rangle & =|70,1,1 / 2,3\rangle \\
|H\rangle & =|490,1,0,6\rangle \\
\left|d_{21}^{0}\right\rangle & =\left|21,3^{*}, 0,2\right\rangle \\
\left|d_{21}^{1}\right\rangle & =|21,6,1,2\rangle \\
\left|d_{15}^{0}\right\rangle & =|21,6,0,2\rangle \\
\left|d_{15}^{1}\right\rangle & =\left|21,3^{*}, 1,2\right\rangle \\
\left|P_{\left.\bar{Q}_{\text {suud }}\right\rangle}\right\rangle & =|210,3,0,4\rangle \\
\left|P_{\bar{Q} \text { suud }}^{\prime}\right\rangle & =\left|105^{\prime}, 3,0,4\right\rangle
\end{aligned}
$$


The pentaquark $P_{\bar{Q} \text { suud }}$ is classified in the 210 of $S U(6)$ to optimize the hyperfine interaction. We also define the state $P_{\bar{Q}}^{\prime}$ suud in the $105^{\prime}$ of $S U(6)$. It is convenient also to define the state $\left|d_{15}^{0} ; d_{21}^{0}\right\rangle$ of two spin-zero diquarks with one diquark classified in the symmetric 21 and the other in the antisymmetric 15 dimensional representation of the color-spin $\mathrm{SU}(6)$ and the analogous state $\left|d_{15}^{1} ; d_{21}^{1}\right\rangle$ of two spin-one diquarks. These two states are orthogonal linear combinations of the two states $|210,3,0,4\rangle$ and $\left|105^{\prime}, 3,0,4\right\rangle$.

We use the simplified form of the color-spin hyperfine interaction [26] commonly applied to systems containing only quarks and no active antiquarks:

$$
V=-(v / 2)\left[C_{6}-C_{3}-(8 / 3) S(S+1)-16 N\right]
$$

where $v$ is a parameter defining the strength of the interaction, $C_{6}$ and $C_{3}$ denote the eigenvalues of the Casimir operators of the $S U(6)$ color-spin and $S U(3)$ color groups respectively, Thia interaction (WW7) is easily evaluated for the states (WW6) by substituting the eigenvalues of the Casimir operators [34,35]:

$$
\begin{aligned}
C_{6}(70) & =66 \\
C_{6}(20) & =42 \\
C_{6}(490) & =144 \\
C_{6}(210) & =(304 / 3) \\
C_{6}\left(105^{\prime}\right) & =(208 / 3) \\
C_{6}(21) & =(160 / 3) \\
C_{6}(15) & =(112 / 3) \\
C_{3}(4) & =(16 / 3) \\
C_{3}(6) & =(40 / 3) \\
C_{3}(8) & =12 .
\end{aligned}
$$

We therefore obtain

$$
V(N)=V(\Lambda)=V\left(d_{21}^{0}\right)=-8 v
$$




$$
\begin{aligned}
V(\Delta) & =8 v \\
V(H) & =-24 v \\
V\left(d_{15}^{0}\right) & =+4 v \\
V\left(d_{15}^{1}\right) & =+(8 / 3) v \\
V\left(d_{21}^{1}\right) & =-(4 / 3) v \\
V\left(P_{\bar{Q} \text { suud }}\right) & =-16 v \\
V\left(P_{\bar{Q} \text { suud }}^{\prime}\right) & =0 \\
V\left(d_{15}^{0} ; d_{21}^{0}\right) & =-4 v
\end{aligned}
$$

where we have noted that the hyperfine interaction vanishes between quarks in a spin-zero diquark and quarks outside the diquark. Thus we can write

$$
\left.\left.V\left(d_{15}^{0} ; d_{21}^{0}\right)=\left|\left\langle d_{15}^{0}\right) ; d_{21}^{0}\right| 210,3,0,4\right\rangle\left.\right|^{2} V\left(P_{\bar{Q} s}\right)+\left|\left\langle d_{15}^{0}\right) ; d_{21}^{0}\right| 105^{\prime}, 3,0,4\right\rangle\left.\right|^{2} V\left(P_{\bar{Q} s}^{\prime}\right)=-4 v
$$

Substituting eqs. (WW10g) and (WW10h) then gives the result already noted in ref. [6].

$$
\begin{aligned}
& \left|\left\langle d_{15}^{0} ; d_{21}^{0} \mid 210,3,0,4\right\rangle\right|^{2}=\left|\left\langle d_{15}^{1} ; d_{21}^{1} \mid 105^{\prime}, 3,0,4\right\rangle\right|^{2}=1 / 4 \\
& \left|\left\langle d_{15}^{1} ; d_{21}^{1} \mid 210,3,0,4\right\rangle\right|^{2}=\left|\left\langle d_{15}^{0} ; d_{21}^{0} \mid 105^{\prime}, 3,0,4\right\rangle\right|^{2}=3 / 4
\end{aligned}
$$

The gain in hyperfine interaction for the $P_{\bar{c} s u u d}$ over the $N D_{s}$ or $\Lambda D$ threshold (degenerate in this symmetry limit) was shown to be equal to the gain for the $H$ over the relevant $\Lambda \Lambda$ threshold and just half the $\Delta-N$ mass splitting.

$$
\begin{aligned}
& M(\Delta)-M(N)=V(\Delta)-V(N)=16 v \\
& B(H)=V(H)-2 V(\Lambda)=-8 v=-(1 / 2)[M(\Delta)-M(N)] \\
& B\left(P_{\bar{c} s u u d}\right)=V\left(P_{\bar{c} s u u d}\right)-V(\Lambda)=-8 v=-(1 / 2)[M(\Delta)-M(N)](W W 13 c)
\end{aligned}
$$

where $\mathrm{B}(\mathrm{X})$ denotes the difference in hyperfine energy between the state $\mathrm{X}$ and the relevant threshold. Thus the $P_{\bar{c} s u u d}$ appeared to be an equally attractive candidate for hyperfine binding as the $\mathrm{H}$ dibaryon [6]. 
The introduction of SU(3) symmetry breaking has been shown to reduce the binding of the $H$ [27], and the strange $P_{\bar{c} s u u d}$ [6]. A similar effect occurs for the doubly-strange $P_{\bar{Q}}$ ssud . This is easily seen by noting that hyperfine binding energy of both the $P_{\bar{Q} \text { suud }}$ and the $H$ is reduced by reducing the color-magnetic interaction of the strange quark. However the strange quark plays no role in the magnetic interactions of the $\Lambda \Lambda, \Lambda D, N D_{s}$ and $\Lambda D_{s}$ final states and their hyperfine binding energies are unaffected by SU(3) symmetry breaking.

It is convenient to write the broken- $S U(3)$ hyperfine interaction in the form for a four quark state in which two quarks of the same flavor are coupled to the 15 of SU(6) and the remaining two quarks with different flavor are coupled to the 21 of $\mathrm{SU}(6)$,

$$
V_{b r}=a_{o} V_{o}+\left(a_{21}-a_{o}\right) V_{21}+\left(a_{15}-a_{o}\right) V_{15}
$$

where $V_{o}$ denotes the values of the hyperfine interaction (WW10i) for the Pentaquark state $|210,3,0,4\rangle$ in the $\mathrm{SU}(3)$ limit for the case where all the quarks have the nonstrange mass $m_{u}$. The coefficient $a_{o}$ is chosen so that the term $a_{o} V_{o}$ contains all the contributions from hyperfine interactions between the two diquarks with the broken-SU(3) masses. The remaining two terms give the correction to the hyperfine interaction within each diquark when the correct masses are used, and the appropriate spin averages defined by the weighting factors (WW12) are used. For the case where the wave function $|210,3,0,4\rangle$ is used,

$$
\begin{aligned}
& \left\langle V_{21}\right\rangle=\frac{3}{4} \cdot V\left(d_{21}^{1}\right)+\frac{1}{4} \cdot V\left(d_{21}^{0}\right)=-3 v \\
& \left\langle V_{15}\right\rangle=\frac{3}{4} \cdot V\left(d_{15}^{1}\right)+\frac{1}{4} \cdot V\left(d_{15}^{0}\right)=+3 v
\end{aligned}
$$

Thus for the case of the doubly-strange $P_{\bar{c} s s u d}$,

$V_{b r}\left(P_{\bar{c} s s u d}\right)=\frac{m_{u}}{m_{s}} \cdot V_{o}+\frac{m_{s}-m_{u}}{m_{s}} \cdot V_{21}-\frac{m_{u}}{m_{s}} \cdot \frac{m_{s}-m_{u}}{m_{s}} \cdot V_{15}=(1-\delta) V_{o}+\delta V_{21}-(1-\delta) \delta \cdot V_{15}$

where

$$
\delta \equiv \frac{m_{s}-m_{u}}{m_{s}}
$$


is a parameter defined [27] to express the suppression of the strange quark hyperfine interaction. For our purposes it is sufficient to work to first order in the SU(3)-breaking perturbation $\delta$. Thus

$$
V_{b r}\left(P_{\bar{Q} s s u d}\right) \approx V_{o}-\delta \cdot\left(V_{o}+V_{15}-V_{21}\right)=-16 v+10 \delta v
$$

The case of the doubly-strange $P_{\bar{Q} \text { ssud }}$ is particularly simple because the two quarks in each diquark have the same mass even when $\mathrm{SU}(3)$ is broken and the flavor permutation symmetry within each diquark in conserved by the broken $\mathrm{SU}(3)$ interaction. In the case of the singly-strange $P_{\bar{Q} \text { suud }}$, the two quarks in the symmetric 21 diquark have different masses and the broken $\mathrm{SU}(3)$ interaction can have off-diagonal matrix elements which change the color-spin permutation symmetry of the diquark, and connect the state to a state in which both diquarks are in the color-spin antisymmetric 15. This contribution which introduces a new state outside the two-dimensional Hilbert space defined by the states $|210,3,0,4\rangle$ and $\left|105^{\prime}, 3,0,4\right\rangle$ has been neglected in previous calculations [6]. We follow this example and neglect these off-diagonal elements which in any case introduce contributions higher order in $\delta$. In this approximation the hyperfine interaction between diquarks is given by an average hyperfine interaction..

$$
V_{b r}\left(P_{\bar{Q} \text { suud }}\right) \approx\left(1-\frac{\delta}{2}\right) \cdot V_{o}+\frac{\delta}{2} \cdot V_{15}-\frac{\delta}{2} \cdot V_{21}=V_{o}-\frac{\delta}{2} \cdot\left(V_{o}-V_{15}+V_{21}\right)=-16 v+11 \delta v
$$

in agreement with the previous result [6]. We thus see that the effects of $\mathrm{SU}(3)$ symmetry breaking on the doubly strange pentaquark are roughly equal to those for the singly-strange case.

One must also consider the possibility that the pentaquarks may not be bound but may be observable as low-lying resonances near threshold in the $D_{s} p, D_{s} \Lambda B_{s} p$ or $B_{s} \Lambda$ system. The combinatorial background can be expected to be very large in the $D_{s} p$ or $B_{s} p$ system where many uncorrelated protons can be present in any high energy event. In this case the doubly-strange pentaquark may be a more easily identified candidate than the singly-strange pentaquark. The number of uncorrelated $\Lambda^{\prime} s$ can be expected to be very much less than the 
number of uncorrelated protons. In either case the pentaquark resonance should appear as an enhancement near the lower end of phase space.

\section{DECAY MODES}

If the pentaquark is a loosely bound $D_{s}-p$ or $D_{s}-\Lambda$ molecule, its decay will resemble that of an off-shell $D_{s}$. However, it may also have the structure of a $\bar{Q}$ and the four-quark state (WW6i),

$$
\left|P_{\bar{Q}_{\text {suud }}}\right\rangle=\left|3^{*}, 1 / 2\right\rangle_{\bar{Q}} \otimes|3,0\rangle_{(\text {uuds })}
$$

where we have labeled the states by the quantum numbers $\left|D_{3}, S\right\rangle$ and now included the quantum numbers of the $\bar{c}$. In the spectator model, the decay of the pentaquark is described as

$$
\left|P_{\bar{c} s u u d}\right\rangle \rightarrow\left|3^{*}, 1 / 2\right\rangle_{(\bar{s} \bar{u} d)} \otimes|3,0\rangle_{(\text {uuds })}
$$

There are many ways that this $\left(3^{*}, 3\right)$ color configuration can fragment into a final state of several color-singlet hadrons, including many states which are not reached in the model of an off-shell $D_{s}$ bound to a spectator proton. The lifetime of the $P_{\bar{c} s u u d}$ may therefore be considerably different from that of the $D_{s}$. However, even for decay modes like $p \phi \pi^{-}$and $p K^{* o} K^{-}$which occur in the $D_{s}-p$ model, the ratio of the two branching ratios can be quite different from that for the $D_{s}$. This can be seen by examining the initial state (WW19) in which the $\bar{c} s$ system is not spin zero as in the $D_{s}$ but is a linear combination of zero and one, with a factor of three favoring spin one. It is like a linear combination of $D_{s}$ and $D_{s}^{*}$.

The $D_{s}^{*}$ component can decay into vector-pseudoscalar via s-wave in contrast to the $D_{s}$ decay which is p-wave. We now show that the s-wave decay has an additional spin factor of 3 favoring the $\phi \pi$ mode over $K^{* o} K^{-}$.

The s-wave decay has no orbital angular momentum in the final four-quark state. Thus we need consider only spin couplings. Since the decay is rotationally invariant we can choose the initial state to be polarized with "spin up" for convenience. We now write the color- 
favored $\bar{c} \rightarrow \pi^{-} \bar{s}$ decay to include spinology and the combination with the spectator quark

$$
\left|\bar{c}_{\uparrow}\right\rangle \cdot\left|s_{\uparrow}\right\rangle \rightarrow\left|\pi^{-} \bar{s}_{\uparrow}\right\rangle \cdot\left|s_{\uparrow}\right\rangle=\left|\pi^{-}\right\rangle \cdot\left|\bar{s}_{\uparrow} s_{\uparrow}\right\rangle
$$

Thus, the color-favored $\bar{c} \rightarrow \pi^{-} \bar{s}$ decay at the quark level leads to a unique spin coupling of the $\bar{s}$ with the spectator quark and requires the $s \bar{s}$ state to have the desired spin 1 for the $\phi-\pi$ decay.

The color suppressed $\bar{c} \rightarrow K^{* o} \bar{u}$ decay written to include spinology and the combination with the spectator quark is

$$
\begin{gathered}
\left|\bar{c}_{\uparrow}\right\rangle \cdot\left|s_{\uparrow}\right\rangle \rightarrow \sqrt{\frac{2}{3}} \cdot\left|K_{\uparrow}^{* o} \bar{u}_{\downarrow}\right\rangle \cdot\left|s_{\uparrow}\right\rangle-\sqrt{\frac{1}{3}} \cdot\left|K_{\rightarrow}^{* o} \bar{u}_{\uparrow}\right\rangle \cdot\left|s_{\uparrow}\right\rangle= \\
=\sqrt{\frac{2}{3}} \cdot\left|K_{\uparrow}^{* o}\right\rangle \cdot\left|\bar{u}_{\downarrow} s_{\uparrow}\right\rangle-\sqrt{\frac{1}{3}} \cdot\left|K_{\rightarrow}^{* o}\right\rangle \cdot\left|\bar{u}_{\uparrow} s_{\uparrow}\right\rangle= \\
=\sqrt{\frac{1}{3}} \cdot\left|K_{\uparrow}^{* o}\right\rangle \cdot\left|(\bar{u} s)_{S=0}\right\rangle+\sqrt{\frac{1}{3}} \cdot\left|K_{\uparrow}^{* o}\right\rangle \cdot\left|(\bar{u} s)_{S=1}\right\rangle-\sqrt{\frac{1}{3}} \cdot\left|K_{\rightarrow}^{* o}\right\rangle \cdot\left|\bar{u}_{\uparrow} s_{\uparrow}\right\rangle
\end{gathered}
$$

Here the color suppressed $\bar{c} \rightarrow K^{* o} \bar{u}$ decay at the quark level is seen to lead to spin couplings of the $\bar{u}$ with the spectator quark which give a probability of $(1 / 3)$ for the $u \bar{s}$ state to have the desired spin 0 for the $K^{* o} K^{-}$decay.

The $D_{s}^{*}$ component can also decay purely leptonically into $\mu \nu$ and $e \nu$ without the helicity suppression factor existing for the pseudoscalar $D_{s}$ decay. Since branching ratio for the decay. $\quad D_{s} \rightarrow \mu \nu$ is about $1 \%$, one might expect significantly higher branching ratios for $\left|P_{\bar{c} \text { suud }}\right\rangle \rightarrow p \mu \nu$ and $\left|P_{\bar{c} \text { suud }}\right\rangle \rightarrow p e \nu$ if the pentaquark has the structure (WW19) of a $\bar{c}$ and a four-quark state (WW6i),

\section{CONCLUSION AND ACKNOWLEDGEMENT}

This paper is contributed to a Memorial Volume for Carl Dover, who had always been interested in searches for exotic hadrons and whom I joined in an investigation of the physics of $\mathrm{H}$ dibaryon searches [36]. I should like to acknowledge many stimulating discussions with

the members of the Tel Aviv experimental group [10,32 and in particular Danny Ashery, 
Sharon May-Tal Beck, Gilad Hurvits, Jechiel Lichtenstadt and Murray Moinester. The results of the pentaquark search in the Fermilab E791 Collaboration should soon be available |11] and hopefully indicate the directions for future searches with better vertex detectors and particle identification. 


\section{REFERENCES}

[1] Harry J. Lipkin, in Hadrons, Quarks and Gluons, Proceedings of the Hadronic Session of the XXIInd Rencontre de Moriond, Edited by J. Tran Thanh Van, Editions Frontieres, Gif Sur Yvette - France (1987), p.691

[2] Harry J. Lipkin, In The Elementary Structure of Matter, Proceedings of the Workshop, Les Houches, France, 1987 Edited by J.-M. Richard et al, Springer-Verlag (1987) p.24

[3] Harry J. Lipkin, in Hadron '87, Proceedings of the Second International Conference on Hadron Spectroscopy, KEK Tsukuba, Japan, edited by Y. Oyanagi, K. Takamatsu and T.Tsuru, KEK Report 87-7 (1987), p.363.

[4] Harry J. Lipkin, In Proceedings of PANIC '87, XI International Conference on Particles and Nuclei, Nucl. Phys. A478, 307c (1988)

[5] Harry J. Lipkin, Phys. Lett. 195B, (1987) 484

[6] C. Gignoux, B. Silvestre-Brac and J. M. Richard, In The Elementary Structure of Matter, Proceedings of the Workshop, Les Houches, France, 1987 Edited by J.-M. Richard et al, Springer-Verlag (1987) p.42; Phys. Lett. B193 (1987) 323

[7] Harry J. Lipkin, In Proceedings of the International Symposium on The Production and Decay of Heavy Flavors, Stanford (1987) Edited by Elliott D. Bloom and Alfred Fridman, Annals of the New York Academy of Sciences, Vol. 535 (1988) p.438

[8] Harry J. Lipkin, In Proceedings of the Topical Conference on Nuclear Chromodynamics, Argonne National Laboratory, May (1988) Edited by J. Qiu and D. Sivers, World Scientific, Singapore (1988) p. 260

[9] Harry J. Lipkin, in Proceedings of the Rheinfels Workshop 1990 on Hadron Mass Spectrum, St.Goar at the Rhine, Germany, Sept. 3-6, 1990, Nucl. Phys. B(Proc. Suppl.) 21 (1991) 258

[10] S. May-Tal Beck, (for FNAL E791 Collab.) In Proceedings of the 1994 Annual Meeting of the Division of Particles and Fields, Albuquerque, N.M., S. Seidel, Ed., World Scientific, (1995) 1177

[11] E.M. Aitala et al., FERMILAB-Pub-97/118-E and to be published

[12] Harry J. Lipkin, In Intersections Between Particle and Nuclear Physics, Proc. Conf. on The Intersections Between Particle and Nuclear Physics, Lake Louise, Canada, 1986 Edited by Donald F. Geesaman AIP Conference Proceedings No. 150, p. 657

[13] E. M. Levin and L. L. Frankfurt, Zh. Eksperim. i. Theor. Fiz.-Pis'ma Redakt (1965) 105; JETP Letters (1965) 65

[14] H.J. Lipkin and F. Scheck, Phys. Rev. Lett. 16 (1966) 71

[15] G. Alexander, H.J. Lipkin and F. Scheck, Phys. Rev. Lett. 17 (1966) 412

[16] Harry J. Lipkin, Physics Letters B335 (1994) 500

[17] Ya. B. Zeldovich and A.D. Sakharov, Yad. Fiz 4 (1966)395; Sov. J. Nucl. Phys. 4 (1967) 283

[18] I. Cohen and H. J. Lipkin, Phys. Lett. 93B, (1980) 56

[19] Harry J. Lipkin, Phys. Lett. B233 (1989) 446; Nuc. Phys. A507 (1990) 205c

[20] A. De Rujula, H. Georgi and S.L. Glashow, Phys. Rev. D12 (1975) 147

[21] Harry J. Lipkin, Nucl. Phys. A478, (1988) 307c

[22] Igal Talmi, Phys. Lett. 205B, (1987) 140

[23] A. Arima, K. Yazaki and H. Bohr, Phys. Lett. 183 (1987) 131

[24] H.J. Lipkin, Phys. Lett. 198B (1987) 131 
[25] H.J. Lipkin, Phys. Lett. 45B (1973) 267

[26] R. L. Jaffe, Phys. Rev. Lett. 38, (1977) 195

[27] J. L. Rosner, Phys. Rev. D 33 (1986) 2043

[28] P. MacKenzie and H. Thacker, Phys. Rev. Letters 65, 2539 (1985)

[29] H. Thacker, private communication

[30] John Weinstein and Nathan Isgur, Phys. Rev. Lett. 48 (1982) 659; Phys. Rev. D27 (1983) 588

[31] Harry J. Lipkin, Phys. Lett. 124B (1983) 509

[32] M. A. Moinester, D. Ashery, L. G. Landsberg and H. J. Lipkin, Zeitschrift fur Physik A 356 (1996) 207

[33] N. Isgur and H. J. Lipkin, Phys. Lett. 99B, 151 (1981).

[34] W. G. McKay and J. Patera, Tables of Dimensions, Indices, and Branching Rules for Representations of Simple Lie Algebras, (Marcel Dekker, New York, 1981) p. 98

[35] H. Högaasen and P. Sorba, Nucl. Phys. B145 (1978) 119

[36] Murray A. Moinester, Carl B. Dover and Harry J. Lipkin, Phys. Rev. C46 (1992) 1082 